\title{
Atomistic simulation study of tensile deformation in bulk nano- crystalline bcc iron
}

\author{
YUAN FuPing* \\ State Key Laboratory of Nonlinear Mechanics, Institute of Mechanics, Chinese Academy of Sciences, Beijing 100190, China
}

Received March 29, 2012; accepted June 16, 2012; published online July 17, 2012

\begin{abstract}
In the present work, the mechanical properties of bulk nanocrystalline (NC) bcc Fe under tensile deformation have been studied by molecular dynamics (MD) simulations. Average flow stress was found to decrease with grain refinement below 13.54 $\mathrm{nm}$, indicating a breakdown in the Hall-Petch relation. A change from grain boundary (GB) mediated dislocation activities to GB activities may be a possible explanation of the breakdown in the Hall-Petch relation. The results also indicate that the average flow stress increases with increasing strain rates and decreasing temperatures. Stress induced phase transformations were observed during the tensile deformation of $\mathrm{NC} \mathrm{Fe}$, and such phase transformations were found to be reversible with respect to the applied stress. The maximum fraction of the $\mathrm{cp}$ atoms was also found to increase with increasing applied stress. Significant phase transformation occurred in the stacking fault zone due to dislocation activities for large grain size (13.54 nm), while significant phase transformation occurred in the GBs due to GB activities for small grain size (3.39 nm). At deformation temperature of $900 \mathrm{~K}$ and above, no apparent phase transformation occurred because all atoms at GBs and grain interior could easily rearrange their position by thermal activation to form local vacancies/disordered structures rather than ordered close packed (cp) structures.
\end{abstract}

molecular dynamics simulation, phase transformation, dislocation activities, grain boundary diffusion, iron

PACS number(s): 61.46.Hk, 64.70.Nd, 81.07.Bc, 62.20.-x, 46.35.+z

Citation: Yuan F P. Atomistic simulation study of tensile deformation in bulk nanocrystalline bcc iron. Sci China-Phys Mech Astron, 2012, 55: 1657-1663, doi: $10.1007 / \mathrm{s} 11433-012-4830-6$

\section{Introduction}

Nanocrystalline (NC) metals (grain size $<100 \mathrm{~nm}$ ) have been the subject of considerable interest due to many unique mechanical properties, such as increased strength/hardness, improved toughness and enhanced diffusivity compared to coarse grained counterparts [1,2]. The strength/hardness has been found to increase with decreasing grain size down to a critical value (10-20 nm), following the well-known HallPetch relation [3,4]. The increased strength/hardness has been attributed to the increased area fraction of grain boundaries (GB), which act as strong barriers to dislocation

*Corresponding author (email: fpyuan@lnm.imech.ac.cn) motions. However, atomistic simulations, theoretical and experimental work show that the strength was found to decrease with further grain refinement below the critical value $(10-20 \mathrm{~nm})$ for fcc metals [5-9], suggesting a shift in the deformation mechanisms from dislocation activities to GB activities.

Atomistic simulations have been shown to be a powerful tool of studying mechanical behavior and deformation mechanisms for NC materials $[10,11]$. These simulations have provided intensive valuable insights into the deformation mechanisms of $\mathrm{NC}$ materials, but most of research has been focused on the fcc metals [5,6,12-17]. Therefore, it is necessary to conduct further investigations [18-21] on the mechanical properties and plastic deformation mecha- 
nisms for bulk NC bcc metals, because of their engineering and technological importance.

As for bec metals such as Fe, phase transformation due to stress or temperature from the ferromagnetic body-centered cubic (bcc) into nonferromagnetic close-packed (cp) structures (fcc $+\mathrm{hcp}$ ), and vice-versa, are of great interest since they are the origin for many important properties of $\mathrm{Fe}$ [22]. The solid to solid phase transformation of $\alpha \rightarrow \varepsilon$ in Fe under shocking loading above $13 \mathrm{GPa}$ was firstly reported by Bancroft et al. [23]. Following this observation, research by theoretical and experimental techniques has been focused on the possibility of the same transformation $(\alpha \rightarrow \varepsilon)$ under static pressure and dynamic shock loading [24-27]. Both molecular dynamics (MD) simulation and in-situ $x$-ray diffraction have confirmed this transformation $(\alpha \rightarrow \varepsilon)$ with shock wave traveling along [001] direction in Fe single crystals [28-30]. However, for shock waves along other single crystal directions and for polycrystalline $\mathrm{Fe}$, both $(\alpha \rightarrow \varepsilon)$ and $(\alpha \rightarrow \gamma)$ transformations were observed, and

the fraction of the fcc phase depended on the shock strength and grain orientation [22,31]. Using MD simulations, [001] oriented nanowires were found to exhibit preferably an $\alpha \rightarrow \gamma$ transformation following Nishiyama-Wassermann path, while [011] and [111] oriented nanowires exhibited a preferred $\alpha \rightarrow \varepsilon$ transformation [32]. The reversibility of the transformation in the elastic regimes and pseudoelasticity were also found by MD simulations for Fe nanowires [33]. Although the solid-solid phase transformations induced by tensile deformation were observed in $\mathrm{Fe}$ nanowires, further studies are still needed to investigate the mechanical properties and phase transformations for bulk $\mathrm{NC} \mathrm{Fe}$ under tensile deformation at high strain rates.

In the present study, MD simulations were used to investigate the mechanical behaviors and deformation mechanisms of bulk NC bcc Fe under tensile deformation. One of the goals of this work was to obtain a more comprehensive understanding of grain size effect on the deformation behaviors and stress-induced microstructural evolutions of bulk NC Fe, thus a wide range of grain size ( $d$ from 3.39 to $27.08 \mathrm{~nm}$ ) was used. Special focus was on the strain rate and temperature effects on the mechanical behaviors and phase transformations for bulk NC Fe.

The paper is organized as follows: the simulation methodology is introduced, including construction of the assembly of bulk NC Fe, boundary conditions, and the interatomic potentials of Fe. The simulations results and discussions are presented, including comparisons with experimental work and other MD simulation results. Finally, we summarize our critical findings on the simulation results.

\section{Simulation methods}

In the present work, atomistic simulations of uniaxial tensile deformation of $\mathrm{NC}$ bcc Fe were performed. Similar to the configuration used by Yamakov [16], all samples contained 6 hexagonally shaped columnar grains with identical size. The columnar axis was [001], and periodic boundary conditions were used in all three directions. In this configuration, only high-energy, asymmetric tilt GBs, each with a misorientation angle of $30^{\circ}$, were produced. MD simulations reported in this work were performed using large-scale atomic/molecular massively parallel simulator (LAMMPS) developed by Plimpton [34]. The time step was 2 fs during the simulations. Before tensile loading, the samples were first subjected to energy minimization by the conjugate gradient method, then gradually heated up to the desired temperature in a step-wise fashion, and finally relaxed in the Nose/ Hoover isobaric-isothermal ensemble (NPT) under both the pressure 0 bar and the desired temperature for $100 \mathrm{ps.} \mathrm{A}$ snapshot of the columnar microstructure with $13.54 \mathrm{~nm}$ grain diameter (defined as the circle encompassing the hexagon) after relaxation at 0 bar and $1 \mathrm{~K}$ is given in Figure 1, including the global orientations of each grain and the direction of the loading. After equilibration processes, the samples were gradually deformed to a maximum elongation of $18 \%$ in the $x$-direction. Pressures in the directions orthogonal to the tensile axis were set to 0 by NPT ensemble during the deformation, leading to a uniaxial loading condition. The local atomic arrangement of the deformed configuration was then visualized using a common neighbor analysis algorithm [35].

The Fe-Fe interatomic forces were calculated based on an embedded atomic method (EAM) potential [36]. This potential was developed by fitting to the first principle forces obtained in a model liquid configuration and also to the other experimentally obtained material properties (lattice parameter, elastic constant, and phase transformation energy). This potential was also verified by Chaussidon et al. [37] as successfully describing the non-degenerate core structure of a screw dislocation, in good agreement with the dislocation structure calculated based on density functional

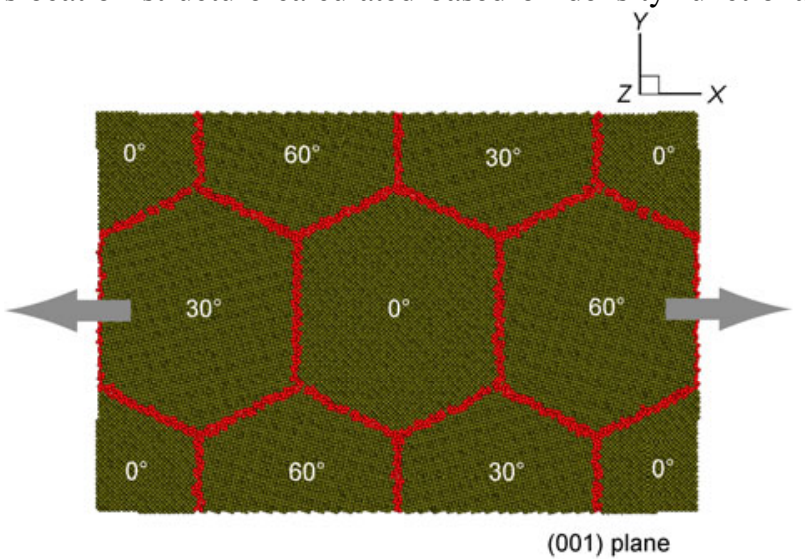

Figure 1 (Color online) Snapshot of the six-grain, columnar microstructure with a grain diameter of $13.54 \mathrm{~nm}$ after relaxation at 0 bar and $1 \mathrm{~K}$. The viewing direction is along the [001] columnar axis, and the orientation of each grain with respect to the axis loading is labeled. The simulation cell is periodic in all three directions and contains 20 (001) atomic planes in the out-of-plane columnar direction. 
theory (DFT) [38]. In order to study the grain size, strain rate and temperature effects on the deformation behavior and phase transformation of $\mathrm{NC}$ bcc Fe, several physical parameters were systematically varied, including grain size $(d=3.39,4.42,10.16,13.54,16.93,20.31,27.08 \mathrm{~nm}$, and the corresponding samples contain 13120, 23460, 118720, $211870,330480,476420,847680$ atoms, respectively), strain rate $\left(\dot{\varepsilon}=3.125 \times 10^{7}, 1.25 \times 10^{8}, 5 \times 10^{8}, 2 \times 10^{9} \mathrm{~s}^{-1}\right)$, and temperature $(T=1,300,600,900,1200 \mathrm{~K})$.

\section{Result and discussion}

In this section, the simulated results for grain size, strain rate and temperature effects on the deformation behavior of bulk $\mathrm{NC} \mathrm{bcc} \mathrm{Fe}$ are addressed respectively.

\subsection{Grain size effect on the deformation behavior of $\mathrm{NC} \mathrm{bec} \mathrm{Fe}$}

Figure 2(a) shows the stress strain curves of bulk NC Fe with various grain sizes. The simulations were performed at temperature of $1 \mathrm{~K}$ and strain rate of $5 \times 10^{8} \mathrm{~s}^{-1}$. As shown in Figure 2(a), the elastic modulus of $\mathrm{NC} \mathrm{Fe}$ decreased with decreasing grain size. This is due to the smaller relative density with smaller grain size [18]. For example, the initial density values of the samples relative to that of single crystal Fe were approximately $99.6 \%$ and $97.8 \%$ for $\mathrm{NC} \mathrm{Fe}$ with grain sizes of 27.08 and $3.39 \mathrm{~nm}$, respectively. Tensile stress was observed to increase with strain up to a certain peak stress, before gradually decreasing to a relatively steady-state value regardless of grain size. These peak stresses are known to be amplified (stress overshoot) due to the high strain rates generally employed in MD simulations $[5,16,17]$. In order to clarify the effect of grain size on the deformation of NC metals using MD simulations, it is a more precise physical meaning to compare the average flow stress over a certain inelastic strain interval. In view of this, average stress for $0-12 \%$ inelastic strain vs. inverse square root of grain size $\left(d^{-1 / 2}\right)$ were plotted in Figure 2(b). These average flow stresses were found to increase to a maximum value of $7.8 \mathrm{GPa}$ with decreasing grain size down to 16.93 $\mathrm{nm}$, but then to decrease with further grain refinement. The usual Hall-Petch relation can be observed for grain size larger than $16.93 \mathrm{~nm}$. Although the trend is not strictly following an inverse Hall-Petch relation, NC Fe becomes softer when the grain size is smaller than $13.54 \mathrm{~nm}$, which is close to the transition point of $14.7 \mathrm{~nm}$ as observed for $\mathrm{NC}$ $\mathrm{Fe}$ by Jeon et al. [19]. In previous research work, the conventional Hall-Petch relation was also reported to break down below grain sizes of 10-15 nm for $\mathrm{NC} \mathrm{Cu}$ [5,8], 10 $\mathrm{nm}$ for $\mathrm{NC} \mathrm{Al} \mathrm{[6],} \mathrm{and} \mathrm{14-20} \mathrm{nm} \mathrm{for} \mathrm{NC} \mathrm{Ni} \mathrm{[7,9].} \mathrm{The} \mathrm{sof-}$ tening effects below the critical grain size were explained by a shift in the deformation mechanism from dislocation activities to GB activities, such as GB diffusion and sliding
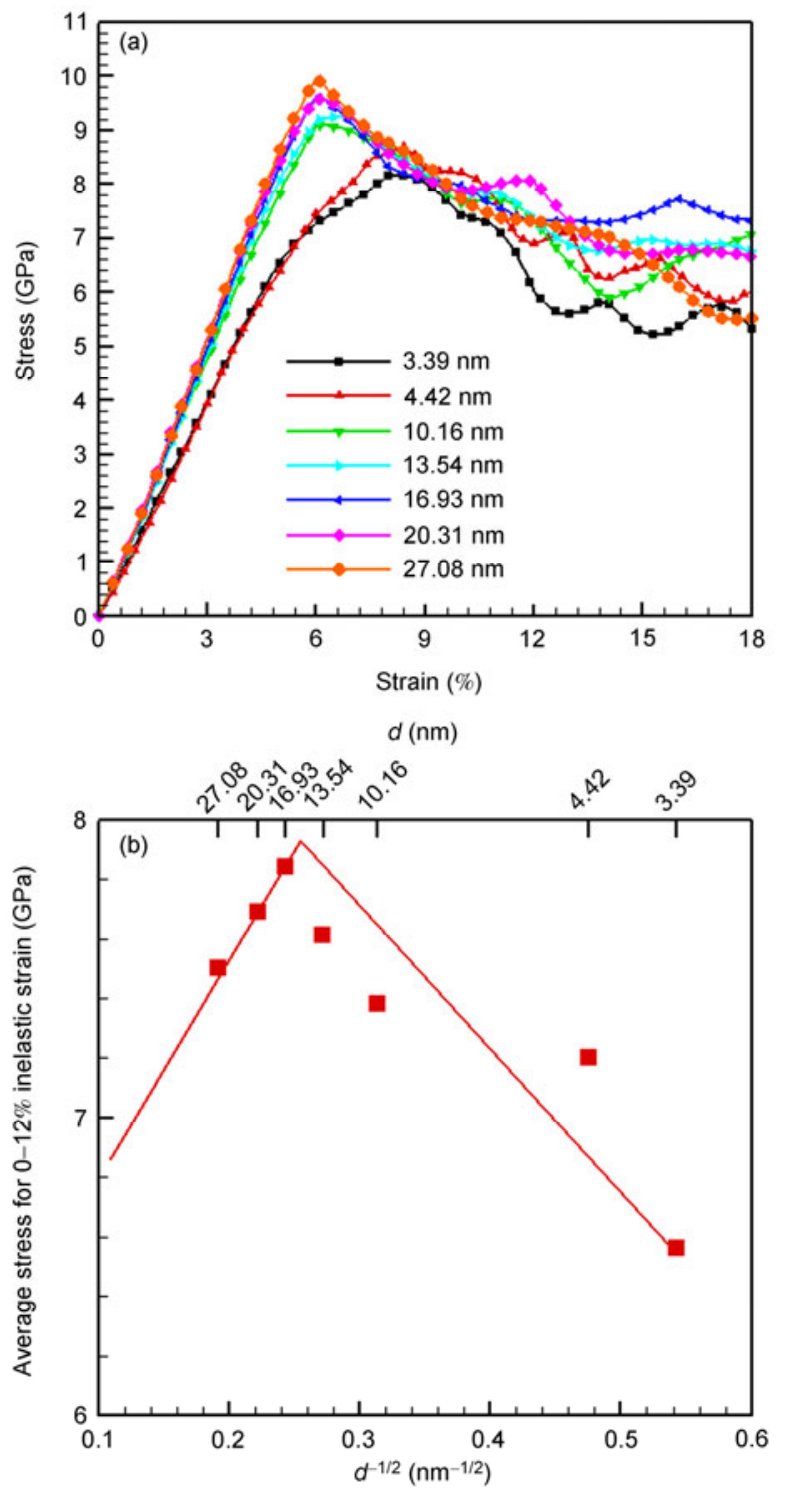

Figure 2 (Color online) (a) True stress-true strain curves of NC Fe with various grain sizes; (b) average flow stress vs. inverse square root of grain size $\left(d^{-1 / 2}\right)$. The solid line represents linear regression fit for simulation data.

\section{[5-9,39].}

We present observations regarding phase transformations induced by the tensile strain in bulk NC Fe in Figures 3-5. Figure 3 shows fraction of $\mathrm{cp}$ atoms (fcc+hcp) as a function of tensile strain for various grain sizes of $\mathrm{NC} \mathrm{Fe}$. The cp clusters consist of mostly fcc structure and only a small portion of hcp structure, which will be clearly shown in the deformed configuration in Figures 4 and 5. The appearance and evolution of the cp clusters as a function of the tensile strain are clearly uncovered by Figure 3. The appearance of the cp clusters begins at a strain of $6 \%$ for each grain size, which corresponds to the onset of plasticity. After onset of plasticity, the fraction of $\mathrm{cp}$ atoms increases with increased strain. However, a peak fraction exists for each grain size, 


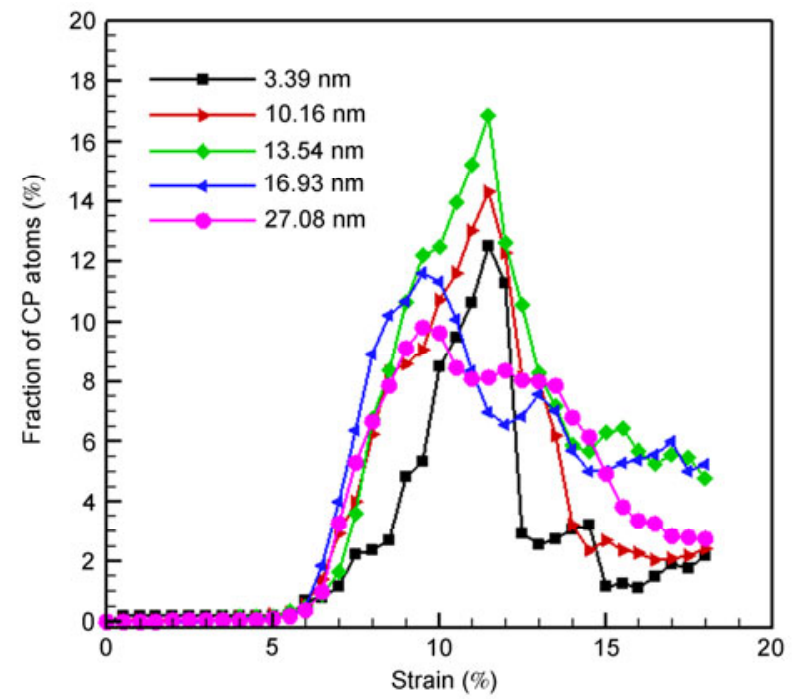

Figure 3 (Color online) Fraction of close packed atoms (fccthcp) as a function of tensile strain for various grain sizes of NC Fe. The deformations is performed at $1 \mathrm{~K}$ and $5 \times 10^{8} \mathrm{~s}^{-1}$.

and the fraction of cp atoms starts to decrease after attaining the peak value. The mechanism of this trend is closely related to the unloading of the specimen, implying that stressinduced phase transformations in NC bcc metals are reversible according to the applied stress [18]. Since the fcc and hcp structures are thermodynamically unstable compared with the bcc structure, the reversibility of the transformation are supposed to be occurring when the flow stress starts to decrease after attaining the peak value.

In order to clearly show the deformation mechanisms for onset of plasticity and evolution of the cp clusters, the deformed configurations as a sequence of tensile strain are shown in Figures 4 and 5 for grain sizes of $13.54 \mathrm{~nm}$ and $3.39 \mathrm{~nm}$ respectively. The color coding of the figure based common neighbor analysis is given in the caption.

Figure 4(a) shows the deformed configuration of 13.54 $\mathrm{nm}$ grain size strained to $6 \%$ elongation, which represents onset of plasticity. Figure 4(b) shows amplified configuration for the rectangular area marked in Figure 4(a). From the Figures 4(a) and 4(b), it is found that partial dislocation emission from GBs and formation of stacking faults on $\{110\}$ plane are responsible for onset of plasticity. In previous work $[40,41]$, it is believed that the atomic mechanism for partial dislocation emission from GBs is due to local atomic shuffling and stress-assisted free volume migration at GBs. It is also interesting to note that slip systems are only activated in the grains with orientation of $0^{\circ}$ at this stage. The reason is that the direction of the maximum shear stress is $45^{\circ}$ to the loading direction, which is consistent with the close packed plane $(\{110\}$ planes $)$ of $0^{0}$ grains. Figure 4(c) shows the deformed configuration of $13.54 \mathrm{~nm}$ grain size strained to $7.5 \%$ elongation. Figure 4(d) shows the amplified configuration for the rectangular area marked

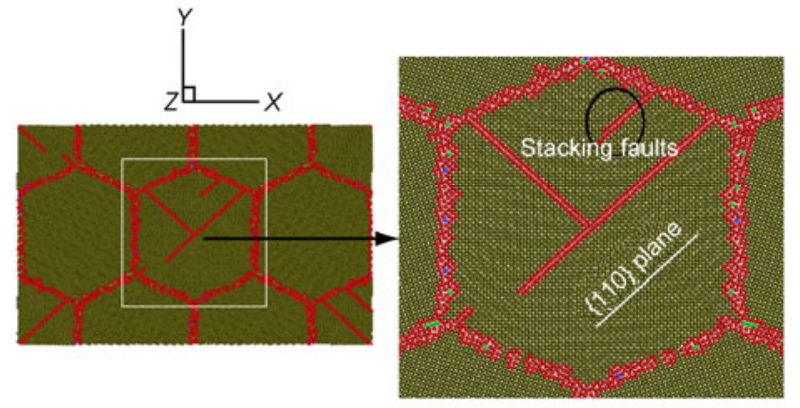

(a)

(b)

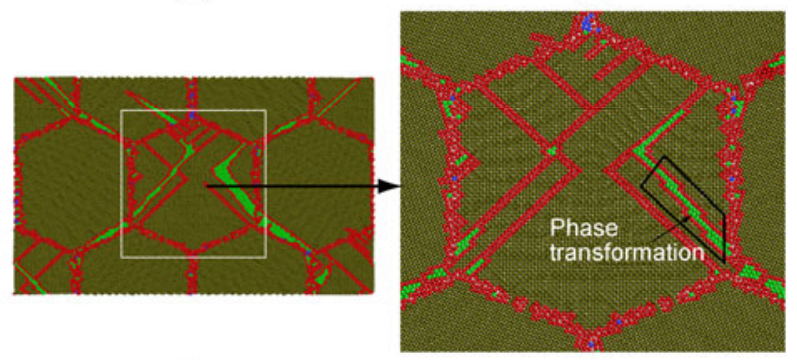

(c)

(d)

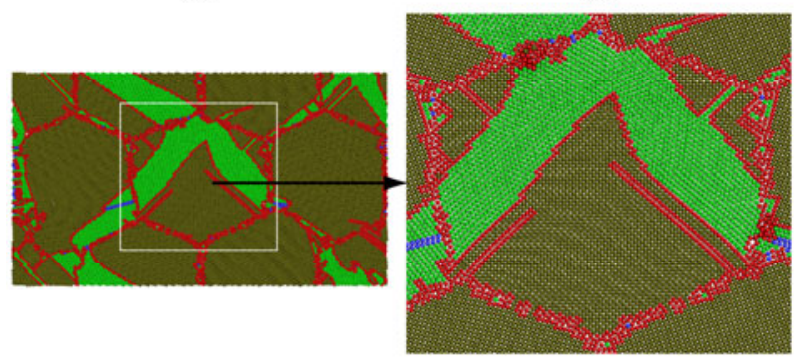

(e)

(f)

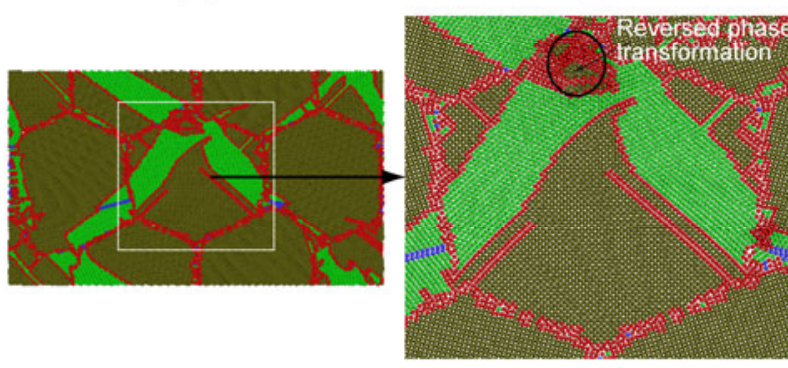

(g)

(h)

Figure 4 (Color online) Deformed configuration of $\mathrm{NC} \mathrm{Fe}$ at tensile strain of (a) $6 \%$ (onset of plasticity); (c) $7.5 \%$; (e) $11.5 \%$; (g) $11.625 \%$. The corresponding amplified configurations for the marked rectangular area are shown in (b) $6 \%$; (d) $7.5 \%$; (f) $11.5 \%$; (h) $11.625 \%$. Golden yellow represents bcc atoms, green is for fcc atoms, blue is for hcp atoms, and red is for all the other atoms, including those of GBS, dislocations and vacancies. The same color coding will be used in the rest of figures. The grain size is $13.54 \mathrm{~nm}$, the deformation temperature is $1 \mathrm{~K}$ and the imposed strain rate is $5 \times 10^{8} \mathrm{~s}^{-1}$.

in Figure 4(c). Significant phase transformation occurs in the stacking fault zone with increased plastic deformation due to dislocation activities. The phase transformation is accommodated by successive emissions of partial dislocation from adjacent planes at GB. The deformed configurations also show that the fraction of $\mathrm{cp}$ atoms first increases 


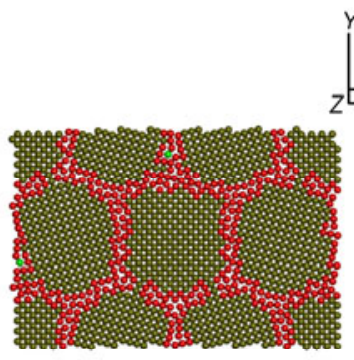

(a)

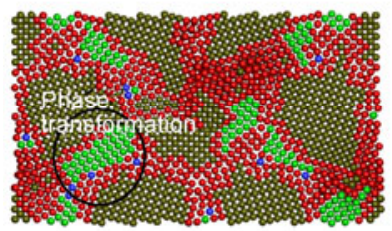

(c)<smiles>[Y][R][X]</smiles>

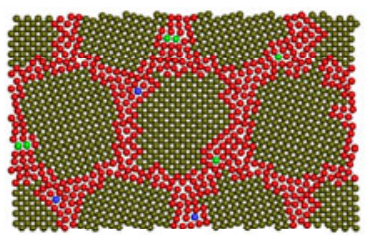

(b)

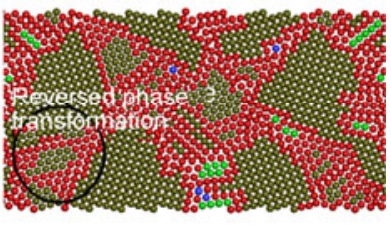

(d)
Figure 5 (Color online) Deformed configuration of $\mathrm{NC} \mathrm{Fe}$ at tensile strain of (a) $0 \%$ (undeformed); (b) $6.0 \%$ (onset of plasticity); (c) 12\%; (d) $18 \%$. The grain size is $3.39 \mathrm{~nm}$, the deformation temperature is $1 \mathrm{~K}$ and the imposed strain rate is $5 \times 10^{8} \mathrm{~s}^{-1}$.

with increased strain, and then reaches the peak value at strain of $11.5 \%$ (Figures 4(e) and 4(f)). The fraction of cp atoms is found to decrease with further plastic deformation after $11.5 \%$, and the reversed phase transformation zone is formed (Figure 4(g) and Figure 4(h)). In the reversed phase transformation zone, some dislocation debris and bcc structures with orientation different from origin grain are both observed. This finding shows that although the phase transformation is reversible, the reversed bcc structure is not necessarily recovered to the origin orientation, indicating local grain rotation induced by the combination process of phase transformation and reversed phase transformation.

Figure 5(a) shows the undeformed configuration of 3.39 nm grain size (strain 0\%), while Figure 5(b) shows the deformed configuration of $3.39 \mathrm{~nm}$ grain size strained to $6 \%$ (onset of plasticity). Deformation mechanisms for onset of plasticity are totally different for this grain size. When the grain sizes become extremely small, the deformation

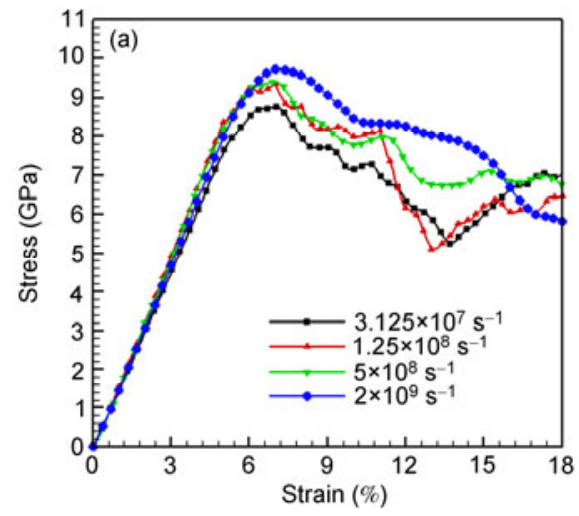

mechanisms clearly shift from GB mediated dislocation activities to GB activities. The fraction of GB atoms significantly increases with increased plastic deformation. Figure 5(c) shows the deformed configuration of $3.39 \mathrm{~nm}$ grain size strained to $12 \%$. The results clearly indicate different mechanisms for phase transformation in two samples with grain sizes of 13.54 and $3.39 \mathrm{~nm}$. Significant phase transformation occurs in the stacking fault zone due to dislocation activities for grain size of $13.54 \mathrm{~nm}$, while significant phase transformation occurs in the GB area due to GB diffusion and sliding for grain size of $3.39 \mathrm{~nm}$. Figure 5(d) shows the deformed configuration of $3.39 \mathrm{~nm}$ grain size strained to $18 \%$. The results also indicate that stress-induced phase transformations for grain size of $3.39 \mathrm{~nm}$ are reversible according to the applied stress.

\subsection{Strain rate and temperature effects on the defor- mation behavior of $\mathrm{NC}$ bec $\mathrm{Fe}$}

Figure 6(a) displays the stress-strain curves for $\mathrm{NC} \mathrm{Fe}$ (grain size $13.54 \mathrm{~nm}$ ) tested at different imposed strain rates at fixed temperature of $1 \mathrm{~K}$, while Figure 6(b) displays the stress-strain curves for $\mathrm{NC} \mathrm{Fe}$ (grain size $13.54 \mathrm{~nm}$ ) tested at various temperatures with a imposed strain rate of $5 \times 10^{8}$ $\mathrm{s}^{-1}$. The results indicate that both the maximum tensile stress and the average flow stress for $0-12 \%$ inelastic strain increase sharply with increased strain rate and decreased temperature. Considering a power-law constitutive relationship, the strain rate sensitivity (SRS) can be written as: $m=\left(\frac{\partial \ln \sigma}{\partial \ln \dot{\varepsilon}}\right)_{T, \varepsilon}$. From Figure 6(a), the SRS for NC Fe (grain size $13.54 \mathrm{~nm}$ ) can be calculated as 0.032 . This value is larger than that of ultra-fine-grained $\mathrm{Fe}$ [42] due to the increased GB mediated plasticity at such small grain size $[18,43]$. The strong temperature dependence of flow stress can be linked to thermal activation processes responsible for the plastic deformation. For example, the activation volume associated with the plastic deformation is proportional to the deformation temperature [18].

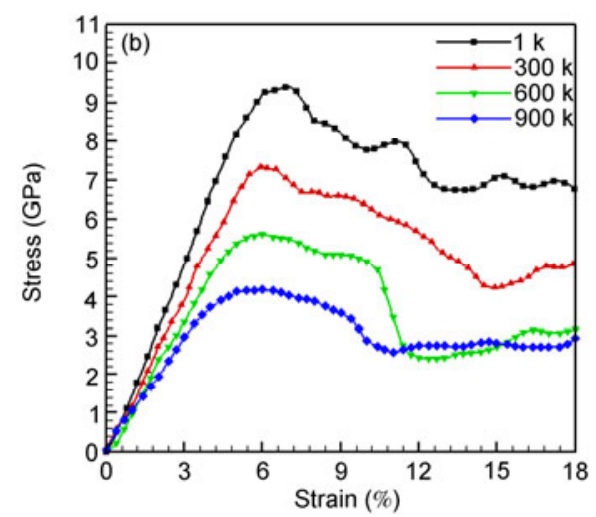

Figure 6 (Color online) (a) True stress-true strain curves of NC Fe tested at various imposed strain rates (the deformation temperature is $1 \mathrm{~K}$ ); (b) true stress-true strain curves of $\mathrm{NC} \mathrm{Fe}$ tested at various temperatures (the imposed strain rate is $5 \times 10^{8} \mathrm{~s}^{-1}$ ). 

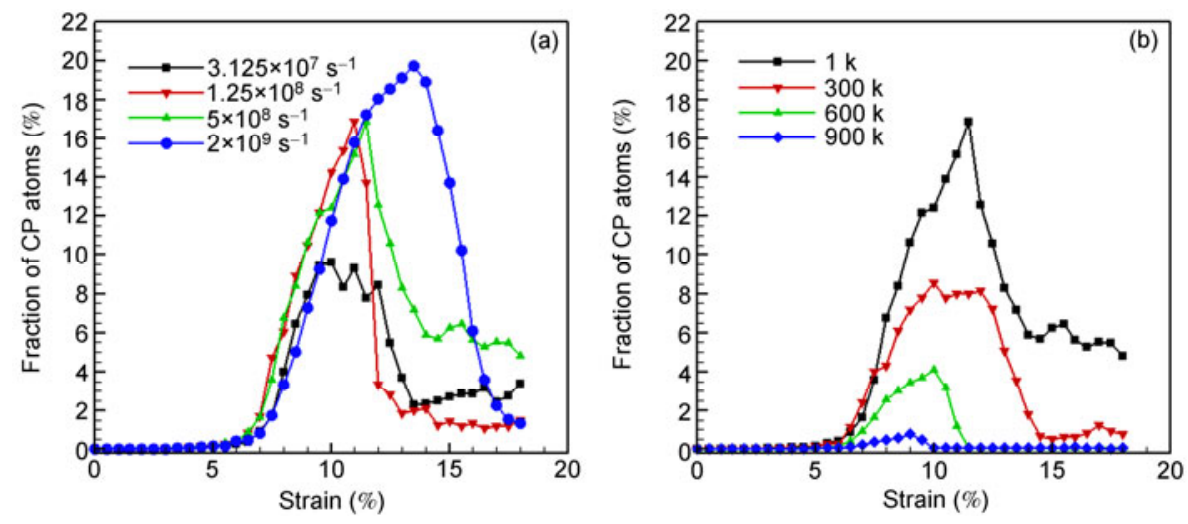

Figure 7 (Color online) (a) Fraction of FCC atoms as a function of tensile strain for various imposed strain rates (the deformation temperature is $1 \mathrm{~K}$ ); (b) fraction of FCC atoms as a function of tensile strain for various temperatures (the imposed strain rate is $5 \times 10^{8} \mathrm{~s}^{-1}$ ).

Figure 7(a) shows the evolution of the cp clusters (fcc+ hcp) with strain at various strain rates. The grain size of the NC Fe sample is $13.54 \mathrm{~nm}$ and the deformation temperature is $1 \mathrm{~K}$. Figure 7(b) shows the evolution of the cp clusters (fcc+hcp) with strain at various temperatures. The grain size of the $\mathrm{NC} \mathrm{Fe} \mathrm{sample} \mathrm{is} 13.54 \mathrm{~nm}$ and the imposed strain rate is $5 \times 10^{8} \mathrm{~s}^{-1}$. Again, the fraction of cp atoms increases with increased strain after onset of plasticity, and decreases after the peak value is attained. The results also indicate that the maximum fraction of the $\mathrm{cp}$ atoms increases with increasing strain rate and decreasing temperature. It is interesting to note that there is no apparent phase transformation when the deformation temperature is above $900 \mathrm{~K}$. At high temperature, dislocation activities disappear due to the higher mobility and diffusivity of atoms. At deformation temperature of $900 \mathrm{~K}$ and above, no apparent phase transformation occurs because all atoms at GBs and in the grain interior can easily rearrange their position by thermal activation to form local vacancies or disordered structures rather than ordered fcc/hcp structures.

From previous results, the fractions of the $\mathrm{cp}$ (fcc+hcp) atoms are closely related to grain size, the imposed strain rate and the deformation temperature in a similar way to that of flow stress. Therefore, certain relationship must exist between the fraction of the cp atoms and the stress condition. This relationship can be clearly observed in Figure 8, which plots the maximum fraction of the $\mathrm{cp}$ atoms against the maximum flow stress. The maximum fraction of the $\mathrm{cp}$ atoms is found to increase with increasing applied stress.

\section{Summary and conclusion}

In the present study, mechanical behaviors and deformation mechanisms of bulk $\mathrm{NC}$ bcc Fe under tensile deformation have been studied by MD simulations. Based on the simulation results, the following concluding remarks are summarized.

The usual Hall-Petch relation can be observed for grain

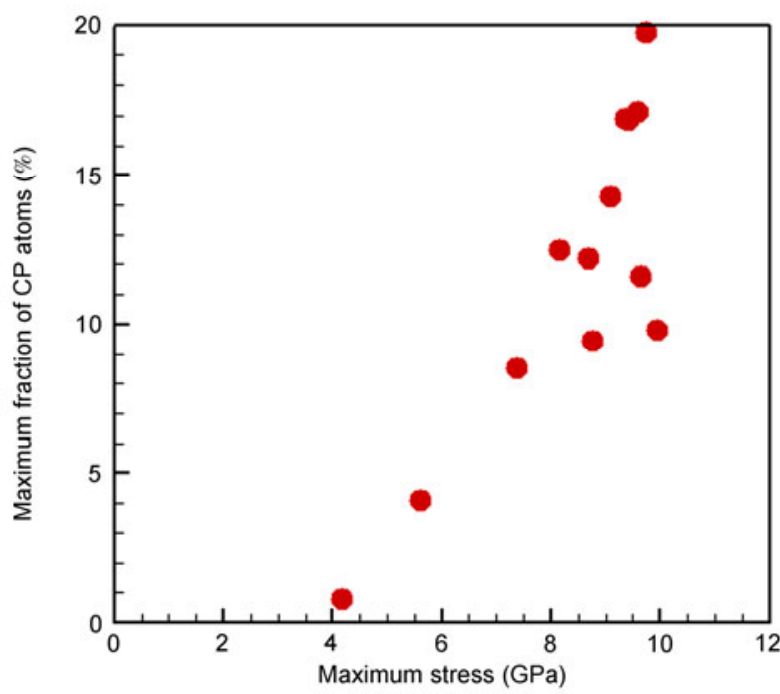

Figure 8 (Color online) Maximum fraction of FCC atoms vs. maximum stress.

size larger than $16.93 \mathrm{~nm}$, while inverse Hall-Petch relation is found for grain size smaller than $13.54 \mathrm{~nm}$. It is found that partial dislocation emission from GBs and formation of stacking faults on $\{110\}$ plane are responsible for onset of plasticity with large grain sizes. However, when the grain sizes become extremely small, the deformation mechanisms clearly shift from GB mediated dislocation activities to GB activities. The results also show that both the maximum tensile stress and the average flow stress for $0-12 \%$ inelastic strain increase with increasing strain rate and decreasing temperature.

Stress induced phase transformations are observed during the tensile deformation of $\mathrm{NC} \mathrm{Fe}$. The fraction of $\mathrm{cp}$ atoms starts to increase with increased strain after onset of plasticity, and then decrease after the peak value. The mechanism of this trend is closely related to the unloading of the specimen, implying that stress-induced phase transformations in $\mathrm{NC}$ bec metals are reversible according to the applied stress. Different mechanisms of phase transformation for different 
grain sizes are also presented. Significant phase transformation occurs in the stacking fault zone due to dislocation activities for grain size of $13.54 \mathrm{~nm}$, while significant phase transformation occurs in the GBs due to GB diffusion and sliding for grain size of $3.39 \mathrm{~nm}$. At deformation temperature of $900 \mathrm{~K}$ and above, no apparent phase transformation occurs because all atoms at GBs and grain interior can easily rearrange their position by thermal activation to form local vacancies or disordered structures rather than ordered fcc/hcp structures. The maximum fraction of the $\mathrm{cp}$ atoms is found to increase with increasing applied stress.

This work was supported by the National Basic Research Program of China (Grant Nos. 2012CB932203 and 2012CB937500) and the National Natural Science Foundation of China (Grants No. 11002151, 10721202 and 11072243).

1 Meyers M A, Mishra A, Benson D J. Mechanical properties of nanocrystalline materials. Prog Mater Sci, 2006, 51: 427-556

2 Dao M, Lu L, Asaro R J, et al. Toward a quantitative understanding of mechanical behavior of nanocrystalline metals. Acta Mater, 2007, 55: 4041-4065

3 Hall E O. The deformation and ageing of mild steel: III discussion of results. Proc Phys Soc B, 1951, 64: 747-753

4 Petch N J. The cleavage strength of polycrystals. J Iron Steel Inst, 1953, 174: 25-29

5 Schiotz J, Jacobsen K W. A maximum in the strength of nanocrystalline copper. Science, 2003, 301: 1357-1359

6 Kadau K, Germann T C, Lomdahl P S, et al. Molecular-dynamics study of mechanical deformation in nano-crystal line aluminum. Metall Mater Trans A, 2004, 35A: 2719-2723

7 Nieh T G, Wang J G. Hall-Petch relationship in nanocrystalline Ni and Be-B alloys. Intermetallics, 2005, 13: 377-385

8 Wei Y J, Gao H J. An elastic-viscoplastic model of deformation in nanocrystalline metals based on coupled mechanisms in grain boundaries and grain interiors. Mater Sci Eng A, 2008, 478: 16-25

9 Wei Y J, Anand L. Grain-boundary sliding and separation in polycrystalline metals: Application to nanocrystalline fcc metals. J Mech Phys Solids, 2004, 52: 2587-2616

10 Li J, Ngan A H W, Gumbsch P. Atomistic modeling of mechanical behavior. Acta Mater, 2003, 51: 5711-5742

11 Mishin Y, Asta M, Li J. Atomistic modeling of interfaces and their impact on microstructure and properties. Acta Mater, 2010, 58: 1117-1151

12 Kumar K S, Van Swygenhoven H, Suresh S. Mechanical behavior of nanocrystalline metals and alloys. Acta Mater, 2003, 51: 5743-5774

13 Schiotz J, Di Tolla F D, Jacobsen K W. Softening of nanocrystalline metals at very small grain sizes. Nature, 1998, 391: 561-563

14 Van Swygenhoven H, Derlet P M, Froseth A G. Stacking fault energies and slip in nanocrystalline metals. Nat Mater, 2004, 3: 399-403

15 Van Swygenhoven H, Derlet P M, Froseth A G. Nucleation and propagation of dislocations in nanocrystalline fcc metals. Acta Mater, 2006, 54: 1975-1983

16 Yamakov V, Wolf D, Phillpot S R, et al. Dislocation processes in the deformation of nanocrystalline aluminium by molecular-dynamics simulation. Nat Mater, 2002, 1: 45-48

17 Li X Y, Wei Y J, Lu L, et al. Dislocation nucleation governed softening and maximum strength in nano-twinned metals. Nature, 2010, 464: 877-880

18 Pan Z L, Li Y L, Wei Q. Tensile properties of nanocrystalline tantalum from molecular dynamics simulations. Acta Mater, 2008, 56: $3470-3480$

19 Jeon J B, Lee B J, Chang Y W. Molecular dynamics simulation study of the effect of grain size on the deformation behavior of nanocrystal- line body-centered cubic iron. Scripta Mater, 2011, 64: 494-497

20 Millett P C, Desai T, Yamakov V, et al. Atomistic simulations of diffusional creep in a nanocrystalline body-centered cubic material. Acta Mater, 2008, 56: 3688-3698

21 Frederiksen S L, Jacobsen K W, Schiotz J. Simulations of intergranular fracture in nanocrystalline molybdenum. Acta Mater, 2004, 52: 5019-5029

22 Kadau K, Germann T C, Lomdahl P S, et al. Shock waves in polycrystalline iron. Phys Rev Lett, 2007, 98: 135701

23 Bancroft D, Peterson E L, Minshall S. Polymorphism of iron at high pressure. J Appl Phys, 1956, 27: 291-298

24 Mao H K, Bassett W A, Takahashi T. Effect of pressure on crystal structure and parameters of iron up to 300 Kbar. J Appl Phys, 1967, 38: $272-276$

25 Giles P M, Longenbach M H, Marder A R. High-pressure alfa-toepsilon martensitic transformation in iron. J Appl Phys, 1971, 42: 4290-4295

26 Bassett W A, Huang E. Mechanism of the body-centered cubic hexagonal close-packed phase-transition in iron. Science, 1987, 238: 780-783

27 Wang F W, Ingalls R, Crozier E D. XAFS study of the bcc-hcp transition of iron. Jpn J Appl Phys Suppl, 1993, 32-2: 749-751

28 Yaakobi B, Boehly T R, Meyerhofer D D, et al. EXAFS measurement of iron bcc-to-hcp phase transformation in nanosecond-laser shocks. Phys Rev Lett, 2005, 95: 075501

29 Kadau K, Germann T C, Lomdahl P S, et al. Microscopic view of structural phase transitions induced by shock waves. Science, 2002, 296 1681-1684

30 Kalantar D H, Belak J F, Collins G W, et al. Direct observation of the alpha-epsilon transition in shock-compressed iron via nanosecond X-ray diffraction. Phys Rev Lett, 2005, 95: 075502

31 Kadau K, Germann T C, Lomdahl P S, et al. Atomistic simulations of shock-induced transformations and their orientation dependence in bcc Fe single crystals. Phys Rev B, 2005, 72: 064120

32 Sandoval L, Urbassek H M. Transformation pathways in the solid-solid phase transitions of iron nanowires. Appl Phys Lett, 2009, 95: 191909

33 Sandoval L, Urbassek H M. Solid-solid phase transitions in Fe nanowires induced by axial strain. Nanotechnology, 2009, 20: 325704

34 Plimpton S. Fast parallel algorithms for short-range moleculardynamics. J Comp Phys, 1995, 117: 1-19

35 Tsuzuki H, Branicio P S, Rino J P. Structural characterization of deformed crystals by analysis of common atomic neighborhood. Comput Phys Commun, 2007, 177: 518-523

36 Mendelev M I, Han S, Srolovitz D J, et al. Development of new interatomic potentials appropriate for crystalline and liquid iron. Phil Mag, 2003, 83: 3977-3994.

37 Chaussidon J, Fivel M, Rodney D. The glide of screw dislocations in bcc Fe: Atomistic static and dynamic simulations. Acta Mater, 2006, 54: 3407-3416

38 Frederiksen S L, Jacobsen K W. Density functional theory studies of screw dislocation core structures in bec metals. Philos Mag, 2003, 83: 365-375

39 Wei Y J, Bower A F, Gao H J. Recoverable creep deformation and transient local stress concentration due to heterogeneous grainboundary diffusion and sliding in polycrystalline solids. J Mech Phys Solids, 2008, 56: 1460-1483

40 Van Swygenhoven H, Derlet P M. Grain-boundary sliding in nanocrystalline fcc metals. Phys Rev B, 2001, 64: 224105

41 Van Swygenhoven H, Derlet P M, Hasnaoui A. Atomic mechanism for dislocation emission from nanosized grain boundaries. Phys Rev B, 2002, 66: 024101

42 Wei Q. Strain rate effects in the ultrafine grain and nanocrystalline regimes-influence on some constitutive responses. J Mater Sci, 2007, 42: 1709-1727

43 Wei Y J, Bower A F, Gao H J. Enhanced strain-rate sensitivity in fcc nanocrystals due to grain-boundary diffusion and sliding. Acta Mater, 2008, 56: 1741-1752 